\title{
Características, epidemiologia e riscos do consumo do narguilé
}

\author{
A narrative review on the characteristics, epidemiology and risks of water pipe \\ consumption
}
Una revisión narrativa sobre las características, epidemiología y riesgos del consumo de narguilé

Lídia Acyole de Souza ${ }^{1 *}$, Marcelo Fouad Rabahi².

\begin{abstract}
RESUMO
Objetivo: Apresentar informações sobre a epidemiologia, características e riscos do consumo do tabagismo no consumo de Narguilé. Revisão bibliográfica: Fumar é considerado como uma enfermidade crónica e é relacionado à ocorrência do câncer, de doenças cardíacas e morte. Nos últimos anos, pesquisadores e governos buscaram juntos combater a pandemia tabagica. Por outro lado, o tabaco continua sendo uma das drogas mais utilizadas por jovens, principalmente quando consumido de forma não convencional. O narguilé é um dispositivo para fumar característico da cultura oriental e que popularizou no ocidente a partir dos anos 90. Estudos em diversos países tem demonstrado prevalências de utilização preocupante, e apontando a juventude como principal grupo de vulnerabilidade para a experimentação. Os riscos do consumo de narguilé vão desde maior exposição a nicotina, monóxido de carbono e outros produtos químicos, à alteração de ritmo cardíaco, doenças contagiosas e canceres. Considerações finais: Informações equivocadas têm servido para mascarar os efeitos nocivos do produto, e que o narguilé pode ser mais prejudicial que o cigarro industrial.
\end{abstract}

Palavras-chave: Tabagismo, Cachimbos de água, Fumar cachimbos de água.

\begin{abstract}
Objective: Present information on the epidemiology, characteristics and risks of tobacco consumption in the consumption of Water pipe. Bibliographic review: Smoking is considered a chronic disease and is related to the appearance of cancers, heart disease and deaths. In recent years, health professionals and governments joined efforts to fight the disease, resulting in a significant decrease of smokers in Brazil. On the other hand, tobacco remains one of the drugs most used by young people, especially when consumed in an unconventional way. The Water pipe is a device for smoking characteristic of Eastern culture and popularized in the West from the 90 studies in several countries have demonstrated disturbing use of prevalence and pointing the youth as the main vulnerable group for experimentation. The risks of Water pipe consumption range from increased exposure to nicotine, carbon monoxide and other chemicals, to changes in heart rate, contagious diseases and cancers. Final considerations: It is concluded that misleading information has served to mask the harmful effects of the product, and that the Water pipe can be more harmful than industrial cigarette.
\end{abstract}

Keywords: Smoking, Smoking water pipes, Water pipe smoking.

\section{RESUMEN}

Objetivo: Presentar información sobre la epidemiología, características y riesgos del consumo de tabaco en el consumo de Narguilé. Revisión bibliográfica: Fumar se considera una enfermedad crónica y está

1 Faculdade Estácio de Goiás (FESGO), Goiânia - GO. *E-mail: lídia.acyole@gmail.com.

2 Universidade Federal de Goiás (UFG), Goiânia - GO. 
relacionada con la aparición de cánceres, enfermedades cardíacas y muertes. En los últimos años, los profesionales de la salud y los funcionarios del gobierno han unido esfuerzos para combatir la enfermedad, lo que resulta en una disminución significativa de los fumadores en Brasil. Por otro lado, el tabaco sigue siendo una de las drogas más utilizadas por los jóvenes, especialmente cuando se consume de manera no convencional. La cachimba es un dispositivo para fumar característico de la cultura oriental y popularizada en Occidente desde los años 90. Los estudios en varios países han demostrado prevalencias de uso preocupante y señalando a los jóvenes como el principal grupo de vulnerabilidad para la experimentación. Los riesgos del consumo de narguile van desde una mayor exposición a la nicotina, el monóxido de carbono y otras sustancias químicas, hasta cambios en la frecuencia cardíaca, enfermedades contagiosas y cánceres. Consideraciones finales: Se concluye que la información errónea ha servido para enmascarar los efectos nocivos del producto, y que la cachimba puede ser más dañina que el cigarrillo industrial.

Palabras clave: Tabaquismo, Pipas de agua, Fumar en pipa de agua.

\section{INTRODUÇÃO}

O tabagismo é uma doença crônica, consequência da dependência a psicológica do consumo de produtos derivados de tabaco, e despertada pela compulsão física da nicotina. Esta doença está associada ao surgimento de outros quadros clínicos graves e tem provocado vultuosos gastos aos cofres públicos, e por isso, ao longo dos anos, nota-se a preocupação de pesquisadores e governantes em estudiosos com esse vício. Essa mobilização tem produzido resultados e pesquisas brasileiras têm apontando queda na prevalência do tabagismo na população adulta e jovem, entretanto é preocupante a quantidade de jovens que ainda experimentam diversas formas de uso do tabaco (BRASIL, 2010; BRASIL, 2013; BRASIL, 2014; BRASIL, 2015.).

Segundo Viegas CBA (2008) existem diferentes formas para a preparação e consumo de um produto de tabaco e é possível categorizá-lo a partir da produção ou não de fumaça. Assim, na categoria de produtos que produzem fumaça, além do cigarro, tem-se o charuto, cachimbo, cigarrilhas, narguilé e o cigarro eletrônico (VIEGAS CBA, 2008).

O narguilé é composto com estruturas que são responsáveis por aquecer o tabaco e resfriar a fumaça produzida por um filtro de água, antes de ser inalada pelo indivíduo. São utilizados mecanismos de filtragem que fazem com que o dispositivo seja equivocadamente tratado como menos nocivo à saúde pelos seus usuários (BRASIL, 2013). O uso do narguilé tem crescido em todo mundo e alertado profissionais da saúde sobre o risco para saúde da população, e grande preocupação é de que este produto seja a iniciação para o tabagismo, aumentando o consumo do cigarro convencional e, principalmente de doenças provocadas pelo hábito. Isso porque, apesar da queda do tabagismo no Brasil, o tabaco ainda é uma das drogas mais experimentadas por adolescentes e jovens (PASUCH C e OLIVEIRA MS, 2014; BARRETO LM, 2011; BRASIL, 2019). O tabagismo nestas fases tem importantes implicações para o bem-estar e para a saúde ao longo da vida, além de aumentar o risco de doenças crônicas não transmissíveis e depressão na vida adulta (BARRETO LM, 2011).

O Instituto Nacional do Câncer (INCA) alerta ainda que a disseminação do narguilé se associa a crença popular de que substâncias tóxicas que o compõe poderia ser filtrada e purificada pela água, eliminando assim seus prejuízos (INCA, 2019). Então, estudos que esclareçam informações de características, riscos e mitos deste produto tornam-se relevantes e emergentes, principalmente no contexto epidemiológico atual. Neste sentido, a presente revisão busca fornecer informações sobre a epidemiologia, características e riscos do consumo do tabagismo no consumo de Narguilé.

\section{REVISÃO BIBLIOGRÁFICA}

\section{Tabagismo no Brasil e no Mundo}

Para a Organização Mundial da Saúde (OMS) o tabagismo é considerado uma doença crônica, epidêmica, difundida por publicidades e propagandas a poderosa indústria do tabaco. É uma das doenças que mais 
levam pessoas a óbito no mundo, e resposável propocionalmente por 6 (seis) de cada 8 (oito) principais causas de morte no mundo (OMS, 2008).

Estimava-se que até o ano de 2.020, cerca de 10 milhões de pessoas viriam a óbito por doenças relacionadas ao hábito de fumar, e a estimativa para 2030 era de 8 milhões de mortes. Entrando, espera-se que além das mortes diretas, as mortes indiretas aumentem essas previsões. Isso porque essa estimativa não previa o surgimento de um vírus contagioso que causaria eventos respiratório.

A COVID-19, doença causada pelo novo coronavirus, surge em 2020 e a OMS e outros órgãos de saúde alertam para o risco do contágio e óbito pela doença em tabagistas passivos e ativos, e consumidores eventuais de derivados de tabaco. Neste novo cenário, o tabagismo pode impactar ainda mais as prevalências de óbitos e nos custos de tratamentos das doenças (SBPT, 2011; INCA, 2020),

Assim, além dos graves problemas promovidos na saúde do indivíduo, o tabagismo promove também um peso econômico considerável para a sociedade ao olhar para custos atrelados a assistência médica e dá à morbidade e mortalidade prematura (PINTO M e UGÁ MAD, 2010). Diretamente, o tabagismo custa quase $\mathrm{R} \$ 57$ no Brasil, dos quais, 39,4 bilhões são gastos com despesas de assistência à saúde e gasto 17,5 bilhões de gastos indiretos devido a perda de produtividade associados ao tabagismo (BRASIL,2013; INCA, 2019).

Estudos nacionais têm mostrado uma queda considerável no tabagismo nos últimos anos. Pesquisas realizadas em 2008, 2013 e 2014 e 2018 já apresentam reduções consideráveis no curto intervalo entre elas (BRASIL, 2010; BRASIL, 2014; BRASIL, 2015; BRASIL, 2019). Entretanto, dados mais expressivos foram apresentados na última década (MONTEIRO, 2007). Isso porque, em 1989 a prevalência de tabagismo era de $34,8 \%$ na população brasileira, apresentado queda de quase $10 \%$ até $2003(22,4 \%)$, chegando, atualmente a $9,3 \%$ entre as capitais.

Por outro lado, um dado que chama atenção nestas pesquisas diz respeito ao tabagismo por faixa etária. Tanto em 2014, quanto em 2018 as maiores prevalências de tabagismo estavam entre pessoas de 25 anos de idade e mais de 55 anos em ambos os sexos (BRASIL, 2015; BRASIL, 2019). Esse resultado permite uma reflexão de que, em um país que demonstra eficiência no combate ao tabagismo nos últimos anos, é preocupante identificar maiores prevalências de tabagismo entre grupos considerados jovens que nasceram em um contexto de leis "antifumo" rígidas, que vão desde a proibição de propaganda e taxação do produto, até a regulamentação de espaços para fumantes.

\section{Narguilé: Origem, epidemiologia e cenário atual}

Existem sugestões de que o Narguilé (waterpipe, Water pipe, Sisha) tenha surgido há mais de 400 anos na Índia. Acredita-se que sua origem tenha sido no reinado do imperador Mongol Akbar (1556-1605), no qual um médico sugeriu que, se o fumo do tabaco passasse pela água antes de ser inalado, seria menos nocivo à saúde humana e a fumaça seria mais pura (MARTINS SE, et al., 2014).

Antes da década de 1990, o uso já era mais frequente na região do Oriente Médio, tornando-se um componente da cultura árabe e de utilização restrita a homens mais velhos. A preocupação com o risco do hábito e estimativas epidemiológicas eram escassas, principalmente pelo baixo monitoramento e preocupação com o tabagismo na região (MAZIAK W, et al., 2015).

O perfil de utilização do narguilé começou a mudar no início dos anos 1990, quando o tabagismo começou a se espalhar entre os jovens. Atualmente, apesar da maior popularidade permanecer em países do Oriente médio e Ásia o uso de narguilé tem crescido nas Américas e na Europa (e tem sido alvo de investigações por estudiosos de todo mundo (CZOLI CD, et al., 2013; ZYOUD SH, et al., 2014; MAZIAK W, et al., 2015; SALLOUM RG, et al., 2016).

As lideranças destas pesquisas são de países ocidentais, entretanto, países em que narguilé é hábito cultural também têm demonstrado preocupação com o uso, e exposto a mesma a partir de dados epidemiológicos (KHAN MSG, et al., 2014). A Adult Tobacco Global (GATS), iniciativa de pesquisa lançada em fevereiro de 2007 pela OMS permite que países levantem dados sobre o uso do tabaco entre adultos e sobre medidas de controle (OMS,2008). 
Pesquisas internacionais da OMS apresentaram a prevalência de consumo de narguilé em diversos países, e colocam Índia (6\%), Egito (3,3\%) e Paquistão (3,0\%) como os principais consumidores, seguidos por Ucrânia (2\%) e Grécia $(1,4 \%)$, enquanto Nigéria $(0,8 \%)$, Malásia $(0,6 \%)$ Indonésia $(0,3 \%)$ mostram prevalências inferiores a $1 \%(\mathrm{WHO}, 2016)$. Por outro lado, na Oceania, uma investigação sobre o uso de narguilé entre imigrantes árabes residentes de Sydney identificaram valores superiores nos quais, mais de $10 \%$ dos respondentes relataram uso diário frequente do dispositivo (CARROLL T, et al., 2008).

Uma pesquisa desenvolvida na Turquia, o país de maiores consumidores de narguilé, analisou os dados de 2008 e 2012 das GATS e identificou que, em 2008, pelo menos 2,3\% da população fazia uso frequente do produto e que a utilização entre os jovens (18 e 26 anos) chegava a 4,3\%. A partir de 2008 as políticas de controle da utilização do produto foram reformuladas e intensificadas, assim, em 2012, prevalência de tabagistas de narguilé chegou a $0,8 \%$ e entre jovens foi de $1,5 \%$, queda superior a $65 \%$ (ERDOL C, et al., 2015).

A América do Norte e na Europa tem apresentado valores alarmantes de utilização de narguilé, principalmente no público jovem. Na cidade de Brent, Noroeste de Londres, uma investigação realizada entre 2399 adolescentes estudantes (maioria do sexo masculino, com idade de 14,5 anos) de 15 escolas secundárias identificou uma prevalência de 7,6\% de uso de narguilé (JAWAD M, et al., 2013).

Pesquisa semelhante realizada na Universidade da Flórida (EUA), com 1203 participantes (idade média $\approx 21.5$ anos; $45,7 \%$ do sexo masculino), a frequência de experimentação foi de $46,4 \%$, e $28,4 \%$ relataram uso continuo no último ano (BARNETT TE, et al., 2013), enquanto outra investigação no país identificou uma prevalência de $3,8 \%$ de uso habitual do narguilé entre 2.528 jovens norte-americanos (SALLOUM RG, et al., 2016).

Um estudo de 4 anos (2006 - 2010) desenvolvido no Canadá com mais de 31.000 estudantes também identificou valores preocupantes quanto ao uso de narguilé. No ano de 2010 , foi encontrada uma prevalência de uso de $10,1 \%$, representando um aumento de $6 \%$ em relação ao ano de 2006 , sendo este significativo entre mulheres, latinos e asiáticos (CZOLI CD, et al., 2013).

Pesquisas realizadas no Brasil também têm comprovado o crescimento da popularidade do narguilé entre jovens, principalmente enquanto forma de primeiro contato com o tabaco (SZKLO AS, et al., 2011; REVELS CC, et al., 2013; MARTINS SE, et al., 2014; BECKERT MC, et al., 2016).

A partir de dados de uma pesquisa nacional com estudantes, Szklo AS, et al. (2011) buscaram analisar a prevalência de consumo do tabaco em três grandes cidades. O consumo de outros produtos do tabaco fumado foi de 18,3\% em Campo Grande, 22,1\% em São Paulo e 4,5\% em Vitória, e o narguilé foi a forma mais popular de consumo do tabaco entre os investigados em todas as cidades.

Os valores encontrados no Brasil não se diferem. Uma investigação pesquisa realizada no Mato Grosso, ao analisar a prevalência e os fatores associados ao uso de narguilé entre 495 adolescente identificou valores tão preocupantes quanto os encontrados no cenário internacional. No estudo foi encontrado que $19,7 \%$ dos participantes fumavam utilizando narguilé, e o consumo do produto aumentava significativamente entre alunos com idades próximas à juventude (16 - 19 anos) (REVELS CC, et al., 2013).

No Paraná, Cristovam MAS, et al. (2013) investigaram o consumo de substâncias psicoativas em uma amostra de escolares. Dentre 242 investigados entre 14 anos -21 anos, 66,4\% relataram ter experimentado narguilé, e dentre os $44,6 \%$ que ainda fazem uso de substâncias psicoativas, $60,1 \%$ consumiram tabaco pelo produto, e se consideram usuários ocasionais.

Martins SE, et al. (2014), em um estudo de coorte prospectiva, analisaram a prevalência do uso de narguilé em alunos de 3 turmas de Medicina em São Paulo, no $3^{\circ}$ e 6ํano do curso. Ao investigar 586 estudantes, a prevalência de uso foi de $47,32 \%$ no terceiro ano e $46,75 \%$ no último ano. Beckert MC, et al. (2016), em um grupo de alunos do curso de Odontologia de Curitiba, identificou 24,94\% de utilização de derivados de tabaco, no qual o narguilé surgiu como principal meio de consumo $(66,23 \%)$, seguido do cigarro industrial $(54,55 \%)$. 
A crescente popularização do narguilé, principalmente entre jovens, pode estar relacionada ao aspecto social que envolve o seu consumo, bem como suas características. O produto tem atraído este público para bares e cafés especializados em todo mundo, e seu consumo é justificado pelo equivocado discurso de que este é menos prejudicial à saúde (WONG LP, et al., 2016).

\section{Características do narguilé e seus riscos para saúde}

Apesar de diversos modelos, o narguilé é composto por peças básicas e apresenta ainda tamanhos e tipos de materiais distinto, mas, em todos os modelos composto por base de água (OLIVEIRA LAS, 2016). No topo do é encontrado o fornilho onde coloca-se o tabaco aromatizado e, por cima dele, o carvão. Ao se aspirar o tubo (mangueira) do narguilé, o ar que passa pelo carvão acende o tabaco, e a fumaça produzida por ele desce pela bomba (corpo) da peça. A fumaça, então, entra na base de vidro e, após entrar em contato com a água, fica mais fria e segue pelo tubo até chegar aos pulmões (OLIVEIRA LAS, 2016).

O produto é rodeado de características que tornam o seu consumo atrativo, e, para a além da aromatização do tabaco, tem-se ainda a aceitação social da cultura de café e restaurantes especializados em narguilé; o auxílio da comunicação em massa e mídias sociais para a sua popularização e a falta de regulamentação de políticas especificas (BRASIL, 2017).

O INCA (2019), reforça essas características ao citar que, um dos grandes responsáveis pelo avanço do narguilé em países ocidentais foram as tecnologias que permitiram a propagação deste produto por possibilitar a comunicação entre os países, e o acesso à cultura oriental. Assim, o aumento da produção do tabaco aromatizado e possibilidade da divulgação por mídias sociais possibilitou não apenas a descoberta do narguilé no mundo, mas também, facilitou o acesso para os países.

Estudos têm buscado apresentar os riscos do narguilé para saúde, e vêm desmistificando que seu consumo seja menos prejudicial em relação ao cigarro industrializado (ABOAZIZA E e EISSENBERG T, 2015; AKHTER S, et al., 2014; ASLAM HM, et al., 2014; KADHUM M, et al., 2014; MEO SA, et al., 2014; BONADIES $\mathrm{N}$, et al., 2013).

No tabaco feito para essa modalidade de fumo é possível encontrar o dobro de nicotina utilizada para o cigarro industrial, estimando-se uma variação de $2 \%$ a $4 \%$, dependendo do fabricante. Além disso, substâncias como alcatrão, benzopireno e arsênio também são encontrados em maior volume na fumaça do narguilé, com o agravante de que o fumante é exposto a maiores volumes de fumaça, quando comparado com o cigarro (VIEGAS CBA, 2010).

Assim como outros produtos derivados do tabaco, a autoadministração do narguilé, somado a quantidade de nicotina em seu composto aumenta os riscos de dependência química, principalmente em contato frequente (ABOAZIZA E e EISSENBER T, 2015).

Uma investigação recente de Akhter S, et al. (2014) realizada com um grupo de Paquistaneses alertou a população quanto à exposição ativa e passiva do narguilé ao $\mathrm{CO}$ (monóxido de carbono). Ao dividir a amostra em 3 grupos (fumantes, fumantes de narguilé e não fumantes) em dois ambientes distintos (espaço com fumantes de narguilé e espaço com fumantes de cigarro).

Akhter S, et al. (2014) identificaram que a exposição ao narguilé aumentou a concentração de CO nos três grupos, e esse aumento também foi considerável ao estar sentado em ambientes de consumo do produto. Em casos mais extremos, seu uso pode provocar envenenamento por excesso de CO (BONADIES N, et al., 2013).

Kadhum M, et al. (2014) buscaram avaliar as alterações cardiovasculares após o consumo do narguilé em 6 (seis) bares Londrinos. Diagnosticaram valores de frequência cardíaca, pressão arterial e concentração de CO após um período de 45 a 90 minutos.

Os autores identificaram alterações significativas nas variáveis cardiovasculares bem como na concentração do $\mathrm{CO}$. As alterações cardiovasculares e de $\mathrm{CO}$ encontradas no estudo foram superiores às 
encontradas em estudos com cigarro convencional. Afirmaram ainda que estas alterações aumentam o risco de hipertensão, e o risco é eminente ainda que o narguilé não seja usado diariamente (KADHUM M, et al., 2014).

Aslam HM, et al. (2014) buscaram identificar o cenário epidemiológico do consumo do narguilé e os efeitos na saúde pública. Encontraram estudos que comprovaram o efeito do narguilé para a disfunção da regulação dos ciclos cardíacos, alteração da frequência cardíaca, risco de contaminação de hepatite e tuberculose (compartilhamento da mangueira), lesões nas cordas e pregas vocais, riscos de câncer semelhante ao do cigarro convencional e alteração nas concentrações de oxido nítrico (ASLAM HM, et al., 2014).

Meo AS, et al. (2014), em um estudo transversal, determinaram os efeitos de fumar narguilé sobre as funções pulmonares e a fração exalada de Óxido nítrico (FeNO) entre sauditas jovens adultos. O estudo identificou que o uso do narguilé provoca reduções significativas na capacidade pulmonar do indivíduo bem como nos níveis de FeNO, e seu uso deve ser considerado nas avaliações clínicas.

Azar RR, et al. (2016) buscou avaliar os efeitos agudos da utilização de narguilé na pressão arterial (PA) e frequência cardíaca ( $F C$ ) em adultos saudáveis. Realizado em 6 restaurantes libaneses, o estudo teve dois grupos de controle: fumantes passivos e não fumantes. Os Fumantes passivos eram indivíduos que estavam sentados em mesas de usuários de narguilé, mas que não fumam tabaco. Não fumantes eram indivíduos que estavam sentados em áreas distintas, em parte de não-fumantes do restaurante.

Os autores concluíram que fumar narguilé por um período de, no mínimo, 15min aumenta significativamente a pressão arterial sistólica e da FC em indivíduos saudáveis, sem doença cardiovascular conhecida. Afirmam ainda que fumar narguilé não deve ser considerado como uma "alternativa" "segura" ao consumo de tabaco (AZAR RR, et al, 2016).

O INCA alerta a notória relação entre o uso de narguilé e o desenvolvimento do câncer de pulmão, doença periodontal (da gengiva), doenças cardíacas, doenças respiratórias (bronquite, enfisema e obstrução das vias aéreas), bem como a tuberculose, herpes e hepatite $C$ causadas pelo compartilhamento da mangueira do produto (BRASIL, 2019).

Os riscos associados ao produto sugerem então a possibilidade de um tratamento, entretanto, vale ressaltar que são raros os estudos associados a temática e que, a forma mais eficiente de intervenção seria a preditiva por meio de ações de educação em saúde e comportamentais (BRASIL, 2019).

Em nota técnica divulgada recentemente (Setembro de 2020) o INCA alertou a população sobre o risco de contaminação pelo novo coronavirus em usuários de Narguilé. O instituto informa que a transmissão pelo compartilhamento de piteira e mangueiras e a preparação manual do produto apresenta-se com alto risco de disseminação da doença, e que, a água contida no jarro é um meio permissivo para a sobrevivência do vírus (INCA, 2020).

\section{CONSIDERAÇÕES FINAIS}

O consumo de Narguilé tem crescido exponencialmente, principalmente em países ocidentais, devido a informações equivocadas a respeito do risco produto e suas características. Assim, o presente artigo reforçou a verdades cientificas em torno do consumo de narguilé, visto que, a grande preocupação é que este sirva como porta de entrada para o cigarro industrializado, e, consequentemente, para as doenças associadas a este. Ademais, espera-se que todo esforço realizado por pesquisadores, profissionais da saúde e autoridades no combate ao tabagismo no Brasil não seja em vão, por isso, estudos relacionados a temática devem ser estimulados e constantemente divulgados.

\section{REFERÊNCIAS}

1. ABOAZIZA E, EISSENBERG T. Waterpipe tobacco smoking: what is the evidence that it supports nicotine/tobacco dependence? Tob Control, 2015; 24: i44-i53.

2. AKHTER S, et al. Comparison of end tidal carbon monoxide (eCO) levels in shisha (water pipe) and cigarette smokers. Tobacco Induced Diseases, 2014; 12(10): 1-4. 
3. ASLAM HM, et al. Harmful effects of shisha: literature review. International Archives of Medicine, 2014; 16(7):1-10.

4. AZAR RR, et al. Acute effects of waterpipe smoking on blood pressure and heart rate: a real-life trial. Inalation toxology, ano 1-4, maio, 2016

5. BARNETT TE, et al. Evidence of emerging hookah use among university students: a cross-sectional comparison between hookah and cigarette use. BMC Public Health, 2013:13:302-312.

6. BARRETO LBM. Comportamentos de risco relacionados a saúde de estudantes universitários. Dissertação (Mestrado em Ciências da Saúde) - Faculdade de Medicina. Universidade Federal do Sergipe, 2011; 73 p.

7. BECKERT MC, et al. Características do uso de produtos derivados do tabaco entre universitários do curso de Odontologia em uma Universidade de Curitiba. Rev Odontol UNESP, 2016; 45(1): 7-14.

8. BONADIES N, et al. When water does not clear the smut from the smoke. BMJ Case Rep, 2013: publish online: 1-4.

9. BRASIL. Instituto Brasileiro de Estatística e Geografia. Pesquisa Nacional em Saúde,2013. Rio de Janeiro: IBGE, 2015.104p.

10. BRASIL. Ministério da Saúde do Brasil. Controle ao tabagismo será ampliado na rede pública de saúde. Brasil.gov, 2013.

11. BRASIL. Ministério da Saúde do Brasil. Vigitel Brasil 2014: vigilância de fatores de risco e proteção para doenças crônicas por inquérito telefônico. Brasilia: MS, 2014. 154p.

12. BRASIL. Ministério da Saúde e Instituto Brasileiro de Geografia e Estatística. Um Panorama da Saúde no Brasil: Acesso e utilização dos serviços, condições de saúde e fatores de risco e proteção à saúde 2008. Rio de Janeiro: MS, 2010. 245p.

13. CARROLL T, et al. Is concern about waterpipe tobacco smoking warranted? Aust N Z J Public Health, 2008; $32(2): 181$ 182.

14. CRISTOVAM MAS, et al. Perfil epidemiológico do uso de substâncias psicoativas por adolescentes. Pediatr Mod., 2013; 49(3): 1-10

15. CZOLI CD, et al. Bidi and Hookah Use Among Canadian Youth: Findings From the 2010 Canadian Youth Smoking Survey. Prev Chronic Dis., 2013;10:1-10.

16. ERDOL C, et al. Waterpipe Tobacco Smoking in Turkey: Policy Implications and Trends from the Global Adult Tobacco Survey (GATS). Int. J. Environ, Res. Public Health, 2015;12:559-566.

17. INSTITUTO NACIONAL DE CÂNCER. Porque aprovar o projeto de Lei do Senado Federal n.2898 de 2019.

18. INSTITUTO NACIONAL DE CÂNCER. Alerta do Instituto Nacional de Câncer José Alencar Gomes da Silva (inca) sobre os riscos do tabagismo e do uso e compartilhamento do narguilé para infecção pelo coronavírus (covid-19). 2020.

19. INSTITUTO NACIONAL DE CÂNCER. Dia nacional de combate ao fumo 2020: tabagismo e coronavírus (covid-19) segunda fase. Disponível em: https://www.inca.gov.br/sites/ufu.sti.inca.local/files//media/document//nota-tecnica-dianacional-de-combate-ao-fumo-coronavirus.pdf. 2020. Acesso em 09 de Out. 2020.

20. INSTITUTO NACIONAL DE CÂNCER. Narguilé: o que sabemos? Rio de Janeiro: INCA, 2019. 100 p.

21. INSTITUTO NACIONAL DE CÂNCER. Uso de narguilé: efeitos sobre a saúde, necessidades de pesquisa e ações recomendadas para legisladores. 2 ed. Rio de Janeiro: INCA, 2017. 49

22. JAWAD M, et al. Prevalence and predictors of water pipe and cigarette smoking among secondary school students in London. Nicotine Tob Res., 2013;15:2069-75.

23. KADHUM M, et al. Measuring the acute cardiovascular effects of shisha smoking: a cross-sectional study. Journal of the Royal Society of Medicine, 2014;5(6): 1-7.

24. KHAN MSG, et al. Shisha..... modern society killer. J Pak Med Assoc., 2014; 64(5): 604-610.

25. Martins SE, et al. Experimentação de e conhecimento sobre narguilé entre estudantes de medicina de uma importante universidade do Brasil. J Bras Pneumol., 2014;40(2):102-110.

26. MAZIAK W, et al. The global epidemiology of waterpipe smoking. Tob Control., 2015;24:i3-i12.

27. MEO SA, et al. Effect of Shisha (Waterpipe) Smoking on Lung Functions and Fractional Exhaled Nitric Oxide (FeNO) among Saudi Young Adult Shisha Smokers. Int. J. Environ. Res. Public Health, 2014; 11: 9638-9648.

28. ORGANIZAÇÃO MUNDIAL DA SAỤDE (OMS). Global Adult Tobacco Survey (GATS), 2008.

29. ORGANIZAÇÃO MUNDIAL DA SAÚDE. Relatório sobre a epidemia global de tabagismo, 2008.

30. PASUCH C, OLIVEIRA MS. Levantamento sobre o uso de drogas por estudantes do ensino médio: Uma revisão sistemática. Cad. Ter. Ocup. 2014; 22(s/n): 171-183.

31. PINTO M, UGÁ MAD. Os custos de doenças tabaco-relacionadas para o Sistema Único de Saúde. Cad. Saúde Pública, 2010; 26(6): 1234-1245.

32. REVELS CC, et al. Factors associated with hookah use initiation among adolescentes. J Pediatr., 2013:89(6):583-587.

33. SALLOUM RG, et al. Waterpipe Tobacco Smoking and Susceptibility to Cigarette Smoking Among Young Adults in the United States, 2012-2013. Prev Chronic Dis., 2016;13(24): 1-9.

34. SOCIEDADE BRASILEIRA DE PENEUMOLOGIA E TISIOLOGIA (SBPT). Tabagismo In: Associação média brasileira; agência nacional de saúde suplementar. Diretrizes clínicas na saúde suplementar, 2011.

35. OLIVEIRA LAS. Experimentação e uso de cigarro eletrônico e narguilé por universitários. Dissertação (Mestrado em Ciências da Saúde) - Faculdade de Medicina. Universidade Federal de Goiás, Goiânia, 2016; 267p.

36. SZKLO AS, et al. Perfil de consumo de outros produtos de tabaco fumado entre estudantes de três cidades brasileiras: há motivo de preocupação? Cad. Saúde Pública, 2011; 27(11):2271-2275.

37. VIEGAS CBA. Formas não habituais de consumo do tabaco. J. bras. Pneumol., 2008; 34(12): 1069-73.

38. WONG LP, et al. Shisha Smoking Practices, Use Reasons, Attitudes, Health Effects and Intentions to Quit among Shisha Smokers in Malaysia. Int. J. Environ. Res. Public Health. 2016; 726(13): 1-14.

39. ZYOUD SH, et al. Bibliometric analysis of scientific publications on waterpipe (narghile, shisha, hookah) tobacco smoking during the period 2003-2012. Tobacco Induced Diseases. 2014; 12(7):1-6. 